\title{
Response to letter: Endoscopic ultrasound-guided confocal endomicroscopy requires high-quality imaging and interpretation for diagnostic evaluation of pancreatic cystic lesions
}

We thank Dr Krishna for commenting on our recent article and summarising the studies of EUS guided nCLE to date. The CONCYST study prospectively recruited patients referred for EUS-FNA of indeterminate $P C L$ to three hepatopancreaticobiliary (HPB) referral centres in the UK. During the study 67 patients were recruited, 56 underwent EUS-nCLE and were included in the final analysis. EUSnCLE findings correlated with final diagnosis (based on imaging, cytology and multidisciplinary team review) in $43 / 56$ (77\%) of cases, compared with $37 / 56$ (66\%) for cytology alone $(P=0.12)$. The overall findings were similar to previous nCLE studies of patients with cystic lesions of the pancreas [1-5] including Dr Krishna's recent US multicentre study (74\% sensitivity, $61 \%$ specificity, $71 \%$ accuracy) [6].

For image interpretation our study used criteria defined by the INSPECT, CONTACT and DETECT studies to identify cyst subtype [1-5]. Sensitivities for individual subtypes in our study ranged between 55 and $100 \%$, some of which were lower than reported by other groups [2]. Dr Krishna proposes that our poorer sensitivities might reflect the suboptimal quality of the images obtained. nCLE images can differ in each cyst subtype depending on the plane the structures are being examined in. Dr Krishna's recent article recognises this variability and highlights that in IPMN papillary epithelial "width" and "darkness" are the most sensitive and accurate criteria for identifying IPMN with highrisk features [7]. The images in Figure 1 were representative of those obtained during our study. Pattern recognition and hence diagnosis in CLE is dynamic and dependent on analysis of multiple images and is not always captured in a single iconic image. Before commencing the study, the investigators underwent online and practical training, visited French centres of excellence to observe procedures and also performed cases together, and liaised with international experts for particular case queries during the study. A clinical advisor was also present during procedures to ensure optimum setup and image registration. With these measures the images in our article were still reflective of those obtained during this study, indicating some of the current challenges to more widespread adoption of this technology. However even within these limitations, in our study nCLE was significantly more accurate at detecting IPMNs than routine cytology ( $90 \%$ vs $69 \%$, p 0.049 ), confirming its utility in a case-by-case basis.

\section{Competing interests}

None

The authors

Margaret G. Keane' ${ }^{1}$, Kofi W. Oppong², Stephen P. Pereira ${ }^{1}$

1 Institute for Liver and Digestive Health, University College London, Royal Free Campus, Pond St, London, NW3 2PF, UK

2 HPB Unit, Freeman Hospital, Newcastle upon Tyne NE7 7DN

Corresponding author

\section{Professor Stephen Pereira}

UCL Institute for Liver and Digestive Health, Royal Free Campus, Pond St, London, NW3 2PF, UK

stephen.pereira@ucl.ac.uk

\section{References}

[1] Giovannini M, Caillol F, Lemaistre A et al. Endoscopic ultrasound guided confocal microscopy: Atlas of cystic pancreatic lesions. Endoscopic ultrasound 2014; 3: (Suppl. 01): S19-21

[2] Napoleon B, Lemaistre AI, Pujol B et al. A novel approach to the diagnosis of pancreatic serous cystadenoma: needle-based confocal laser endomicroscopy. Endoscopy 2015; 47: 26-32

[3] Napoleon B, Lemaistre AI, Pujol B et al. In vivo characterization of pancreatic cystic lesions by needle-based confocal laser endomicroscopy (nCLE): proposition of a comprehensive nCLE classification confirmed by an external retrospective evaluation. Surg Endosc 2016; 30: 2603-2612

[4] Nakai Y, Iwashita T, Park do H et al. Diagnosis of pancreatic cysts: EUS-guided, through-the-needle confocal laser-induced endomicroscopy and cystoscopy trial: DETECT study. Gastrointestinal endoscopy 2015; 81: 1204-1214

[5] Krishna SG, Brugge WR, Dewitt JM et al. Needle-based confocal laser endomicroscopy for the diagnosis of pancreatic cystic lesions: an international external interobserver and intraobserver study (with videos). Gastrointestinal endoscopy 2017; 86: 644-654.e642

[6] Krishna SG, Hart PA, Malli A et al. Endoscopic Ultrasound-Guided Confocal Laser Endomicroscopy Increases Accuracy of Differentiation of Pancreatic Cystic Lesions. Clin Gastroenterol Hepatol 2019; 17 : pii: S1542-3565(19)30648-2

[7] Krishna SG, Hart PA, DeWitt JM et al. EUS guided confocal laser endomicroscopy : prediction of dysplasia in intraductal papillary mucinous neoplasms (with video). Gastrointest Endosc 2019; 19: pii: S00165107(19)32247-3

\section{Bibliography}

DOI http://dx.doi.org/10.1055/a-1038-4398

Endoscopy International Open 2020; 08 : E312

(c) Georg Thieme Verlag KG

Stuttgart · New York

eISSN 2196-9736 